This item was submitted to Loughborough's Research Repository by the author.

Items in Figshare are protected by copyright, with all rights reserved, unless otherwise indicated.

\title{
Development of subliminal persuasion system to improve the upper limb posture in laparoscopic training: a preliminary study
}

\section{PLEASE CITE THE PUBLISHED VERSION}

http://dx.doi.org/10.1007/s11548-015-1198-x

\section{PUBLISHER}

(c) Springer Verlag

\section{VERSION}

AM (Accepted Manuscript)

\section{PUBLISHER STATEMENT}

This work is made available according to the conditions of the Creative Commons Attribution-NonCommercialNoDerivatives 4.0 International (CC BY-NC-ND 4.0) licence. Full details of this licence are available at: https://creativecommons.org/licenses/by-nc-nd/4.0/

\section{LICENCE}

CC BY-NC-ND 4.0

\section{REPOSITORY RECORD}

Zhang, Di, Salvatore Sessa, Weisheng Kong, Sarah Cosentino, Daniele Magistro, Hiroyuki Ishii, Massimiliano Zecca, and Atsuo Takanishi. 2019. "Development of Subliminal Persuasion System to Improve the Upper Limb Posture in Laparoscopic Training: A Preliminary Study”. figshare. https://hdl.handle.net/2134/17596. 


\section{Title}

\section{Development of subliminal persuasion system to improve \\ the upper limbs posture in laparoscopic training: a}

\section{preliminary study}

Authors

Di Zhang ${ }^{1}$, Salvatore Sessa ${ }^{1}$, Weisheng $\mathrm{Kong}^{1}$, Sarah Cosentino ${ }^{1}$, Daniele Magistro ${ }^{2}$, Hiroyuki Ishii ${ }^{1}$, Massimiliano Zecca ${ }^{3,4,5}$, and Atsuo Takanishi ${ }^{6}$

1. Waseda University, 2-2 Wakamatsu-cho, Shinjuku-ku, Tokyo 162-8480, Japan (contact: contact@takanishi.mech.waseda.ac.jp)

2. Torino University, Department of Psychology, Via Verdi 10, 10124, Torino, Italy (contact: danielemagistro@gmail.com)

3. Loughborough University, Loughborough LE11 3TU, United Kingdom (contact: m.zecca@lboro.ac.uk)

4. National Centre for Sports and Exercise Medicine - East Midlands (NCSEM-EM), Loughborough, UK

5. NIHR Leicester-Loughborough Diet, Lifestyle and Physical Activity Biomedical Research Unit (BRU), Loughborough, UK

6. Department of Modern Mechanical Engineering, Waseda University, and Humanoid Robotics Institute, Waseda University, Tokyo, Japan.

Abstract

Purpose: Current training for laparoscopy focuses only on the enhancement of manual skill and does not give advice on improving trainees' posture. However, a poor posture can result in increased static muscle loading, faster fatigue, and impaired psychomotor task performance. In this paper, the authors propose a method, named subliminal persuasion, which gives the trainee real-time advice for correcting the upper limbs posture during laparoscopic training like the expert but leads to a lower increment in the workload.

Methods: A 9-axis Inertial Measurement Unit (IMU) was used to compute the upper limbs posture, and a Detection Reaction Time (DRT) device was developed and used to measure the workload. A monitor displayed not only images from laparoscope, but also a visual stimulus, a transparent red cross superimposed to the laparoscopic images, when the trainee had incorrect upper limbs posture. One group 
was exposed, when their posture was not correct during training, to a short (about $33 \mathrm{ms)} \mathrm{subliminal}$ visual stimulus. The control group instead was exposed to longer (about $660 \mathrm{~ms}$ ) supraliminal visual stimuli.

Results: We found that subliminal visual stimulation is a valid method to improve trainees' upper limbs posture during laparoscopic training. Moreover, the additional workload required for subconscious processing of subliminal visual stimuli is less than the one required for supraliminal visual stimuli, which is processed instead at the conscious level.

Conclusions: We propose subliminal persuasion as a method to give subconscious real-time stimuli to improve upper limbs posture during laparoscopic training. Its effectiveness and efficiency were confirmed against supraliminal stimuli transmitted at the conscious level: subliminal persuasion improved upper limbs posture of trainees, with a smaller increase on the overall workload.

Keywords

Laparoscopy, Subliminal persuasion, Workload, Subliminal visual stimuli, Supraliminal visual stimuli

Introduction

I. Laparoscopic training

Laparoscopy is a practice widely recognized for abdominal operations [1], and its benefits for the patients, compared to open surgery, are well known: net immunologic advantage [2], reduced post-operative pain, faster recovery and decreased perioperative complications [3],[4],[5]. There is also a consistent number of emergency operations that are now performed by laparoscopy [6]. It basically consists in inserting long thin instruments, called laparoscopes, into small incision on the body of the patient; the surgeon then manipulates the laparoscopes by looking at a monitor projecting the images of the body through a video-camera inserted in another small incision, and performs the required operations.

Although for patients there are many advantages, laparoscopic surgery method is much more difficult for doctors, because of the reduced depth perception, affected hand-eye coordination, movement disorientation and reduced haptic feedback. Hence, special training is required to achieve the necessary skill to perform effectively various procedures, for example cutting, knotting or burning within minimal space and without direct visualization. Additionally, these technical difficulties increase the overall workload [7] and forearms fatigue [8] of surgeons.

Different training curricula have been adopted for young and expert surgeons, including exercises on dry boxes, virtual reality and live tissue practices [9]. However, these exercise routines are designed to specifically improve doctors' manual skills, there is no system focusing instead on the doctors' posture. However, poor posture, especially upper limbs posture, in which novice and expert largely differ during 
the laparoscopic training [10], can cause static muscle load and fatigue [11] as well as impaired psychomotor task performance [12]. Normally, trainees always receive advice and feedback from expert doctors on their posture during training. However, posture advice adds up on the very high, both psychological and physical, workload of laparoscopic training, lowering self-esteem and diverting attention from the laparoscopic task [13]. As a result, psychological and physical workload becomes much higher and the performance level decreases. In Fig. 1 the relationship between performance and workload is shown. The level of the workload can be divided in three regions: a low level workload region, Region 1, a middle level workload region, Region 2, and a high level workload region, Region 3 [14]. In these three regions, the relationship curve between performance and workload is very different:

- In Region 1, the workload is too low and the operator's performance does not reach its optimal level

- In Region 2, the workload is nor too low nor excessive. In this region, the operator can reach and maintain a relatively constant and high level performance.

- In Region 3, the operator's workload is excessive. In this region, the workload increases more than the operator's capability of compensation, and performance decreases.

For surgical training, we need to keep the training workload in Region 2, avoiding excess workload that can impair performance.

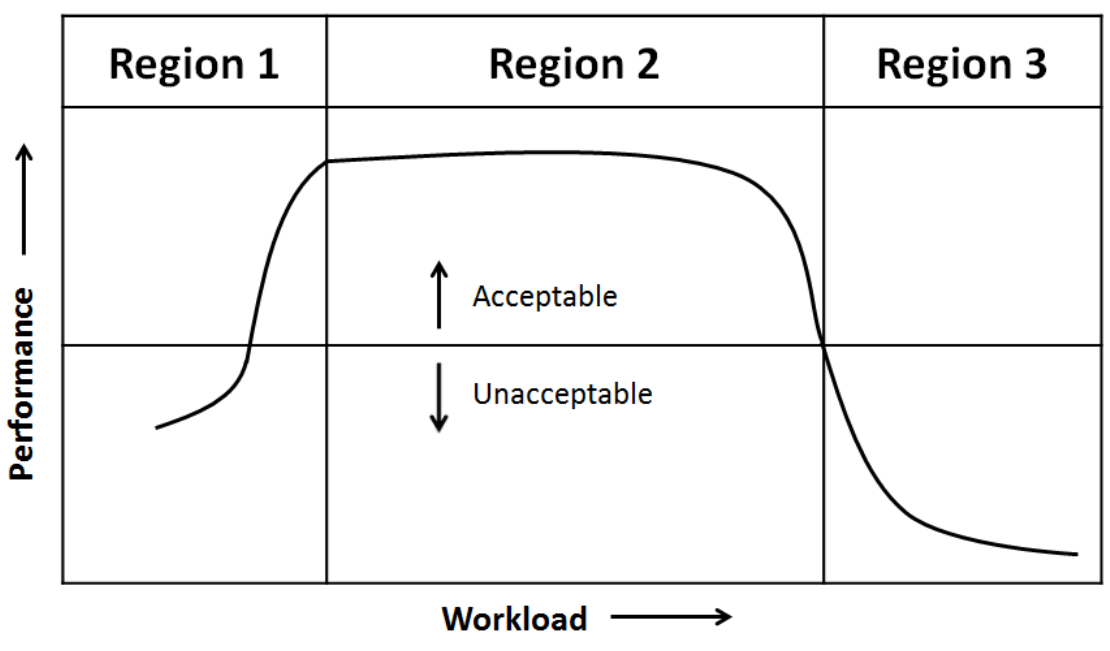

Fig. 1 Performance as a function of operator workload. (From Lysaught et al, 1989)

In this article, we present a method that gives the trainee real time feedback to correct upper limbs posture during laparoscopic training, named subliminal persuasion stimuli, which could help in reducing fatigue and improving performances without adding extra workload.

\section{Subliminal persuasion}

During laparoscopic training, the main task for the trainee is concentrating on the training action. In this study, processing the posture feedback stimulus and correcting upper limbs posture is a subtask. Hence, the feedback stimulus should not distract from the main task and the subtask should avoid further 
increasing of the overall workload as much as possible. However, conscious information processing in humans requires an adequate stimulus to evoke a response or induce a sensation, such as supraliminal stimulus, drawing attention [15]. In this research, we propose to use subliminal persuasion instead of information transferred on the conscious level. Subliminal persuasion is a type of communication that is not intended to be processed consciously but influences thoughts, feelings, and/or behavior at the subconscious level [16] [17]. Subliminal persuasion stimuli can be transmitted and processed through four human perceptive channels [18]:

1) Vision: images transmitted so quickly that they are perceived only subliminally;

2) Audition: sounds inaudible to the conscious mind but hearable and interpretable to the subconscious mind;

3) Olfaction: fragrances so weak that exposed subjects are not conscious of the aroma;

4) Mechanoreception: pressure or vibratory patterns noticeable only at an unconscious level.

Among the four channels listed above, the vision channel is the best candidate for subliminal stimulation during laparoscopic training, as the subliminal stimuli to improve trainee posture can be provided in the same channel of the supraliminal information from the training device, minimizing the overall sensory load.

Therefore, objectives of this paper are:

Obj 1: To develop a real-time visual subliminal persuasion system;

Obj 2: To improve trainee's upper limbs posture during laparoscopic training, and to compare the upper limbs posture correction and additional workload using either subliminal stimuli or supraliminal stimuli.

With this study we want to prove that, during laparoscopic training:

Hyp 1: Subliminal stimuli feedback can correct trainee's upper limbs posture at the same level as supraliminal stimuli feedback;

Hyp 2: Subliminal stimuli feedback has lower impact on the overall training workload compared to supraliminal stimuli feedback.

The structure of the paper is as follows: Materials section introduces the motion capture system used for measuring the upper limbs posture and the new developed device to measure the attention level. Methods section explains the experiment protocol and data analysis. Results and Discussion sections present and elaborate the differences between subliminal stimuli group and supraliminal stimuli group. Conclusions section summarizes the results and proposes the future work.

\section{Materials}

\section{Inertial Measurement Units (IMU) hardware and setup}

Upper limbs posture of the subjects was recorded by one Inertial Measurement Units (IMU) located on the left forearm (Fig. 2). 


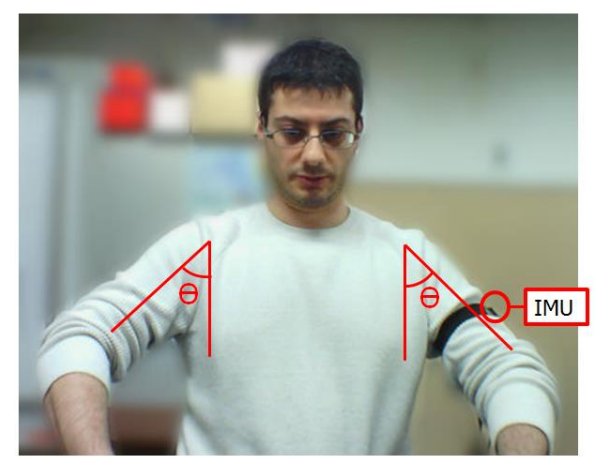

Fig. 2 Hardware setup with IMU

The IMU used for this experiment, named Waseda Bioinstrumentation number 4 Refined (WB-4R), developed by our research team [19], consists in a full inertial system composed by a 3-axis digital accelerometer LIS331DLH from STMicroelectronics, a 3-axis analog gyroscope LYPR540AH from STMicroelectronics and a 3-axis digital compass HMC5843 from Honeywell (Fig. 3). Data are processed by a 32-bit microcontroller STM32F103CBT6 from STMicroelectronics and transmitted to a central board via CAN bus, the central board transmits the grouped data via Bluetooth to a remote PC for data storage and analysis. The small dimensions and the reduced weight has made its use possible in several applications [20].

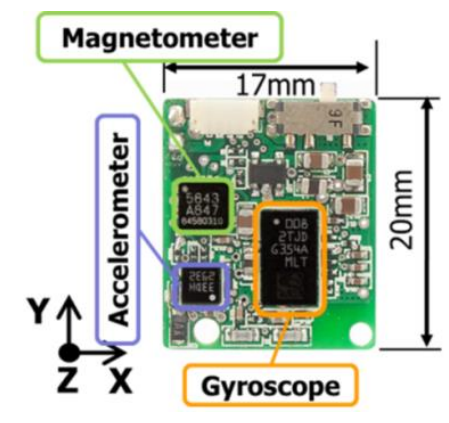

Fig. 3 Sensor side of IMU

\section{Detection Reaction Time (DRT) hardware and setup}

Workload is defined as an intervening variable that quantifies the tuning between the demands of the environment and the capacity of the organism. In our specific setting, workload has an inverse relationship with the level of attention [21]. For this reason, reaction time, a simple and quantifiable parameter to assess the level of attention [22] was measured during the laparoscopic training by a new developed device named Detection Reaction Time (DRT) (Fig. 4). 


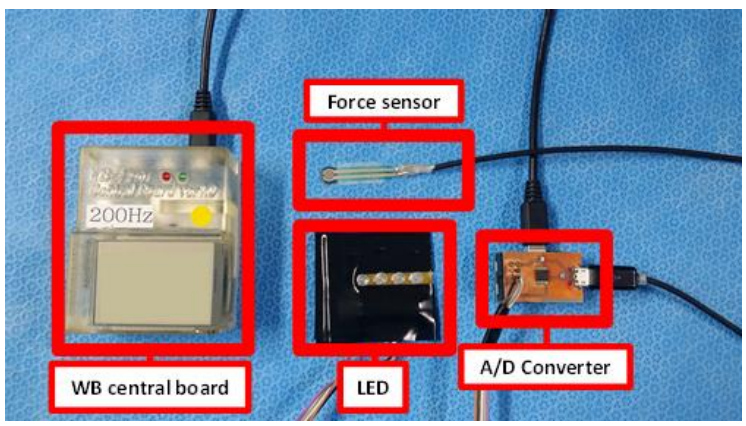

Fig. 4. Hardware setup with DRT

The DRT device is composed by four parts: a force sensor FSR ${ }^{\text {TM }} 400$ Series from Interlink Electronics, a LED OSG58A5111A device from OptoSupply, an A/D converter, and a WB central board. The LED is automatically turned on at random (following a uniform distribution ranging from $10 \mathrm{~s}$ to $25 \mathrm{~s}$ ) and would be turned off by the subject pressing the force sensor. The time elapsed between turning on and turning off, namely the time required to the subject to acknowledge the device signal and react, was recorded and transmitted via CAN bus to the WB central board, where all the DRT and IMU data were synchronized, grouped and sent via Bluetooth to PC for data storage.

\section{Laparoscopic training device}

The training box used in the experiments was Endowork-pro II (MC Medical Inc., Japan). A web camera C615 from Logitech mounted on the training box transmitted a remote image of the operating area on a monitor, placed in front of the training box. The two laparoscopic instruments were the KAR \#26173 by Karl Storz Company.

\section{System setup}

Two computers were used for the experiment (Fig. 5). Computer 1 was used for DRT data acquisition and video recording. Computer 2 displayed images from the training box, analyzed upper limbs posture from IMU data, and generated the posture visual feedback during the training. Using one computer proved in fact to be difficult, due to computational demands of the system: the real-time images were not transmitted accurately and the whole system slowed down.

The LED part of the DRT device was placed on the left upper corner of the monitor and the force sensor was attached on an easy-to-press position on the right laparoscopic instrument. A WB-4R sensor was placed on the left forearm. The DRT and IMU data were collected, stored and analyzed through a GUI. 

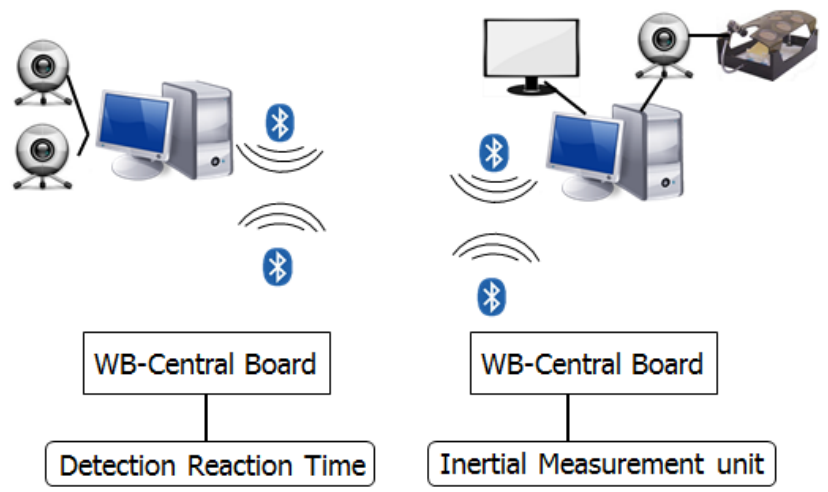

Fig. 5 System Schematic

\section{Methods}

\section{Subjects}

12 participants (10 males, 2 females; 10 right handed, 2 left handed; average age: 25 years) were recruited among laboratory members and all the participants were novices in laparoscopic surgery. The experiments were performed in accordance with the ethical standard defined by the committee of Waseda University and in accordance with the ethical standards laid down in the 1964 Declaration of Helsinki and its later amendments. All the experiments were recorded by the video camera on the training box.

The participants were randomly divided into two groups. One group was named subliminal stimuli group and the control group was named supraliminal stimuli group. Each group had one female participant and one left handed participant.

\section{Preparation}

The preparation consisted in the following sessions:

1. Explanation session: each subject was briefed about the entire procedure, in particular how to use the laparoscopes and how to perform the laparoscopic exercise, including information on the correct movements that should be done and the correct posture to keep during the entire exercise, stressing on the importance of a correct upper limbs posture. For supraliminal stimuli group (Group B), the link between the upper limbs posture and supraliminal feedback was clearly explained, and the trainees were instructed to modify their upper limbs posture if they received the visual stimuli. The subliminal stimuli group (Group A), was not informed about the stimuli.

2. Simple reaction time baseline recording session: the subjects were asked to be fully relaxed, and then to focus on the LED on the left side edge of the monitor. When the LED turned on they needed to press the force sensor as fast as they could. The exercise was repeated 5 times consecutively and the average reaction time was saved as simple reaction time baseline for each subject.

\section{Experiment protocol}

The laparoscopic task to be performed in this experiment is called pipe cleaner. This is a standard task for laparoscopic training, and consists in passing a pipe cleaner through 4 small circles following a set 
procedure involving both arms. The trainee can only see the interior of the laparoscopic training box indirectly, on a monitor, through a web camera located internally in the box. Fig. 6 shows the experimental environment, with the pipe-cleaner platform inside the training box and the monitor. This exercise is widely used in the laparoscopic training, because it enhances the manual skill of the doctor and it is economically affordable [23] [24].

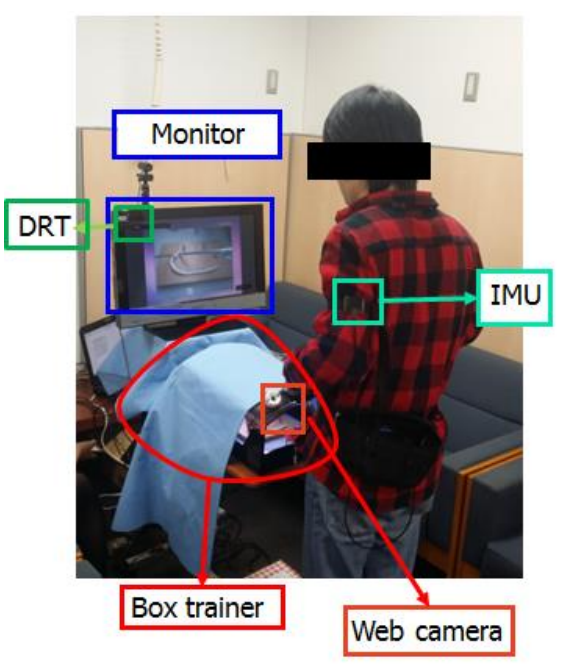

Fig. 6 Experimental environment

After the preparation, the subjects were asked to perform a not recorded trial, to test the system. To remove the influence of sensorimotor dominating left area in some subjects, all the subjects need to pass the pipe cleaner through the small rings using left instrument and catching it on the other side using the right instrument when going from left to right, and in the opposite way when going in the opposite direction. The operative sequence of pipe-cleaner training is showed in Fig. 7. The complete procedure in Fig. 7 denotes one trial for the pipe-cleaner training. In this experiment, each subject executed ten repetitions of that same trial. No time limitation was imposed for the completion of a trial. After every trial, the subject was asked if tired. If the subject was tired, he/she had a 10 min break to ease the effect of the muscle fatigue. During the training, LED of DRT turned on randomly, and the subjects was required to press the force sensor fixed on the laparoscopic instrument as soon as possible to turn off the LED. 


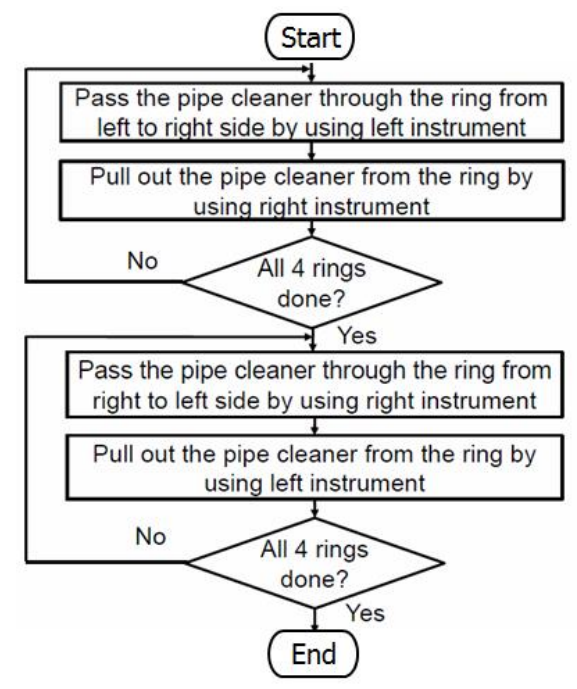

Fig. 7 Operative sequence of pipe-cleaner training

\section{Subliminal stimuli and supraliminal stimuli}

For the experiment, as stated earlier, the subjects have been separated in two groups depending on the type of stimuli. One group received subliminal stimuli (Group A), the other received supraliminal stimuli (Group B).

In a previous study, during laparoscopic pipe-cleaner training, the angle $\theta$ of shoulder, shown in Fig.1, was very different between novice and expert. This angle $\theta$ is the absolute inclination of the arm with respect to the ground: in case of experts, the angle $\theta$ was about 15 degrees [12].

We calculated the angle from the IMU data with the formula (1),

$$
\theta=\sin ^{-1} \frac{\left|A_{z}\right|}{\sqrt{A_{x}^{2}+A_{y}^{2}+A_{z}^{2}}}
$$

where $A_{x}, A_{y}, A_{z}$ are the acceleration along x-axis, y-axis and z-axis respectively.

When the trainee's shoulder angle was more than 20 degree, a big transparent red cross was superimposed to the laparoscopic task images, once every 2 seconds (Fig. 8).

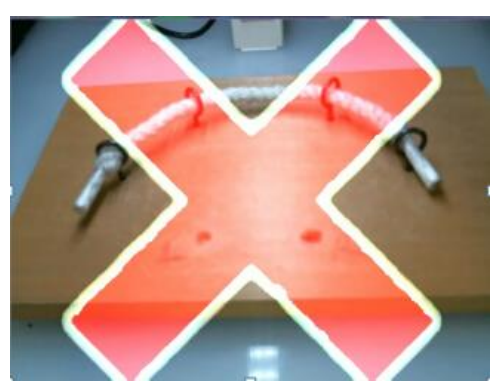

Fig. 8 Red Cross shown on video

The red cross visual feedback was presented in a different way to the two groups:

Subliminal stimuli group (Group A): a subliminal visual signal consists in images transmitted so quickly that a human does not see them consciously but perceives them only at the subconscious level. We set as 
an acceptable exposure time to the red cross, in order to be a subliminal signal, $33 \mathrm{~ms}$.

Supraliminal stimuli group (Group B): a supraliminal visual signal must be consciously perceived and processed. Hence, for this group, the exposure time to the red cross stimulus was $660 \mathrm{~ms}$.

\section{Results and Discussion}

In this study, we focused on three parameters: execution time, number of errors, and training reaction time. These parameters were chosen because they are linked to our research hypotheses. All the significant differences were detected using the t-test.

\section{Execution Time}

Fig. 9 shows the execution time for every trial. For both Group A and Group B, a decreasing trend of execution time was found. Furthermore, in Fig. 10 where the comparison between first and final trial for two groups is shown, it can be seen that for both Group A and Group B, execution time of the first trial and final trial present a significant difference, with $\mathrm{p}<0.01$. Hence, we could say that this laparoscopic pipe cleaner training session was successful, even though we used a modified system with a feedback stimulus to correct the trainee's posture and with an additional subtask to measure the reaction time.

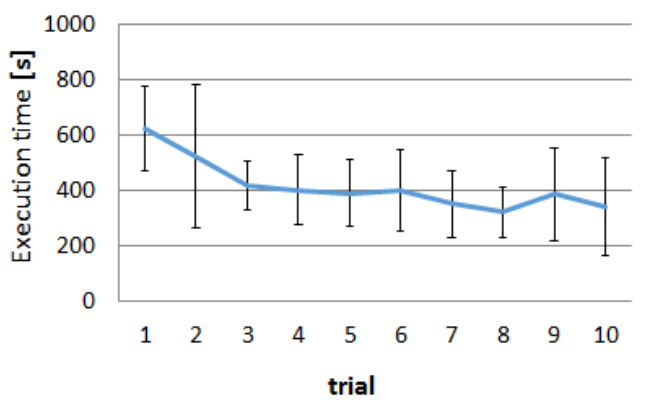

Group A Subliminal visual stimuli

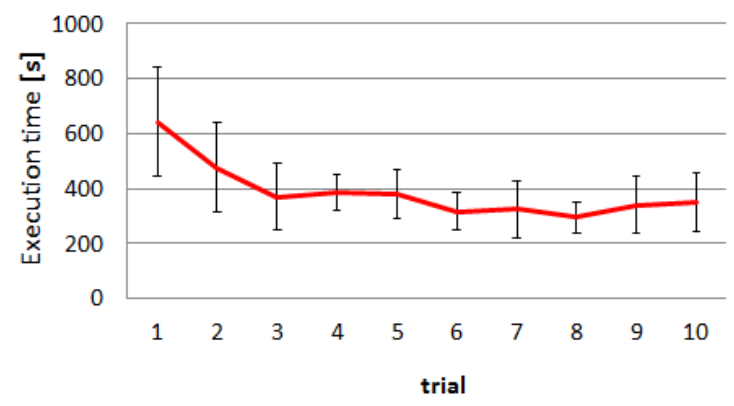

Group B Supraliminal visual stimuli

Fig. 9 Execution time for each group

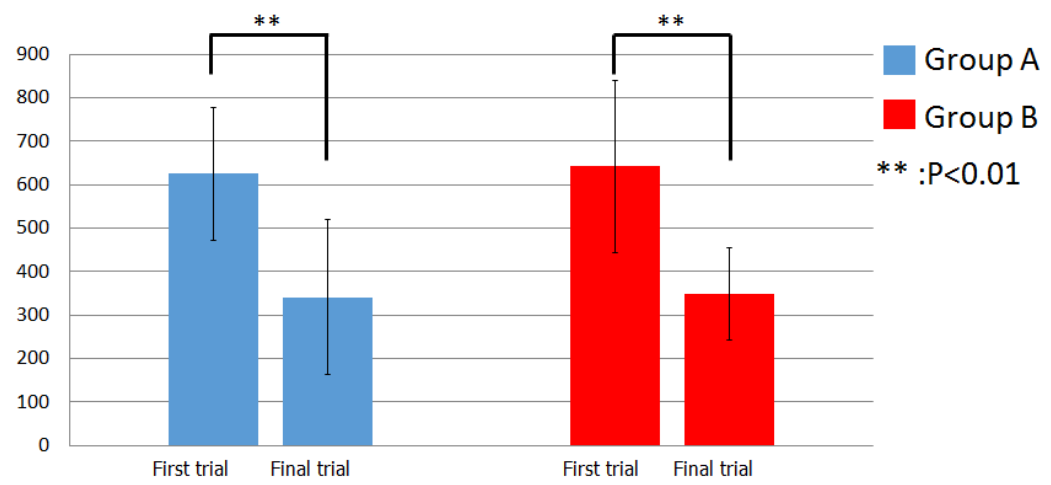

Fig. 10 Comparison between first and final trial

Fig. 11 shows also that the initial skill level for both Group A and Group B was comparable (p=0.815) and that after the training, the final reached skill level for both Group A and Group B is again comparable, 
presents no significant difference $(\mathrm{p}=0.763)$. This is very important, because it means that the different types of stimuli to improve the trainees' upper limbs posture did not affect significantly the laparoscopic training.

As for our second hypothesis, the workload for Group A should be lower than for Group B, therefore the overall performance for Group A should be better than for Group B. However, we did not find any difference between the performances of the two groups in the final trial. This is probably due to the fact that the pipe-cleaner task is not very difficult: so, although in Group B the workload increased, the overall workload was still in Region 2 and thus, was still acceptable (Fig. 1). However, as the training task becomes much more complex and psychologically stressful, we believe that the final performance of the training will be different between both groups.
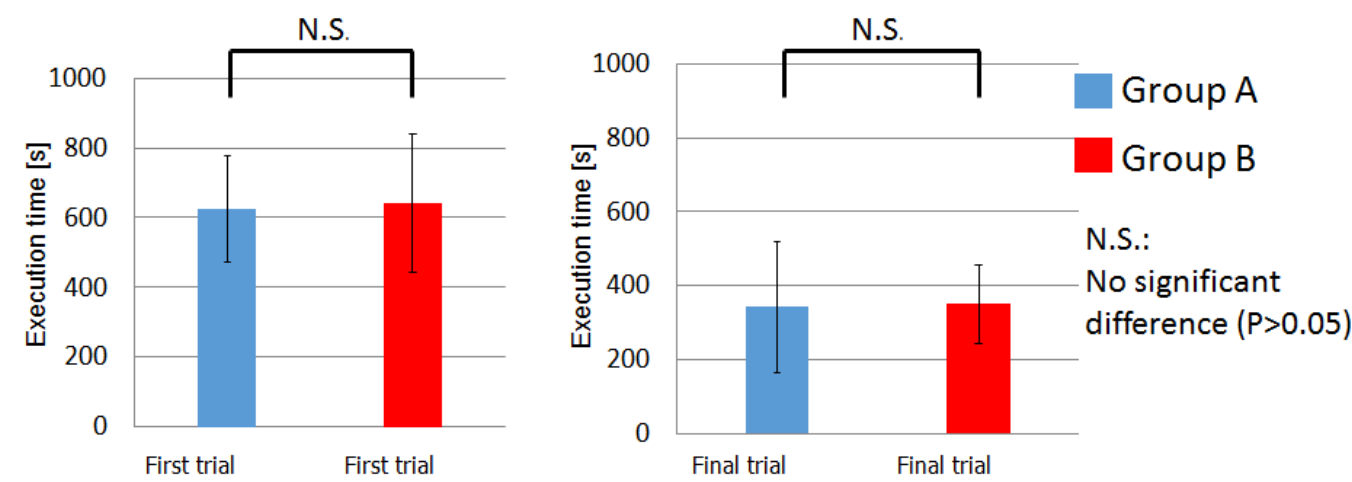

Fig. 11 Comparison between both groups on first trail and final trial

\section{Number of Errors}

During the pipe-cleaner training, when the trainee's upper limbs angle $\theta$ was beyond 20 degrees, this action was recognized as a wrong movement and counted as error once every two seconds.

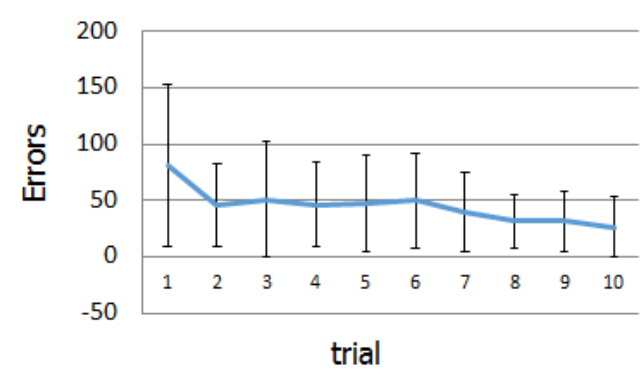

Group A Subliminal visual stimuli

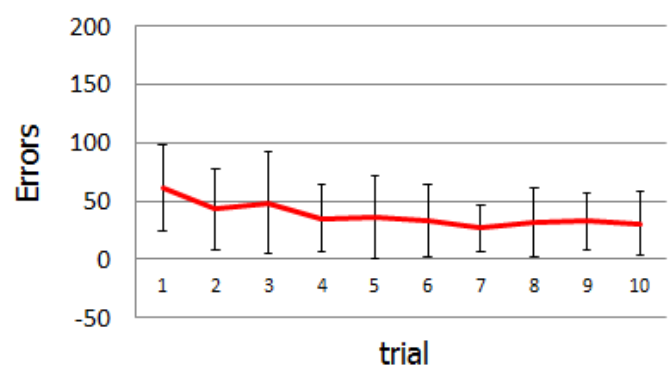

Group B Supraliminal visual stimuli

Fig. 12 Number of Errors for each group

As shown in Fig. 12, we found a decreasing trend for total number of errors per trial from first trial to final trial. Additionally, we can see in Fig. 13, that Group A and Group B number of errors in the first trial was not significantly different ( $\mathrm{p}=0.929)$, as the two groups started at the same level (Fig. 9), but there was instead a significant difference between the first and final trials for each group (Fig. 14), signifying 
that the trainees' upper limbs posture has improved during the training. As we expected, the subliminal persuasion during laparoscopic training worked and these subliminal stimuli affected the trainees' upper limbs posture, having the same effect as the supraliminal stimuli (Hyp 1).

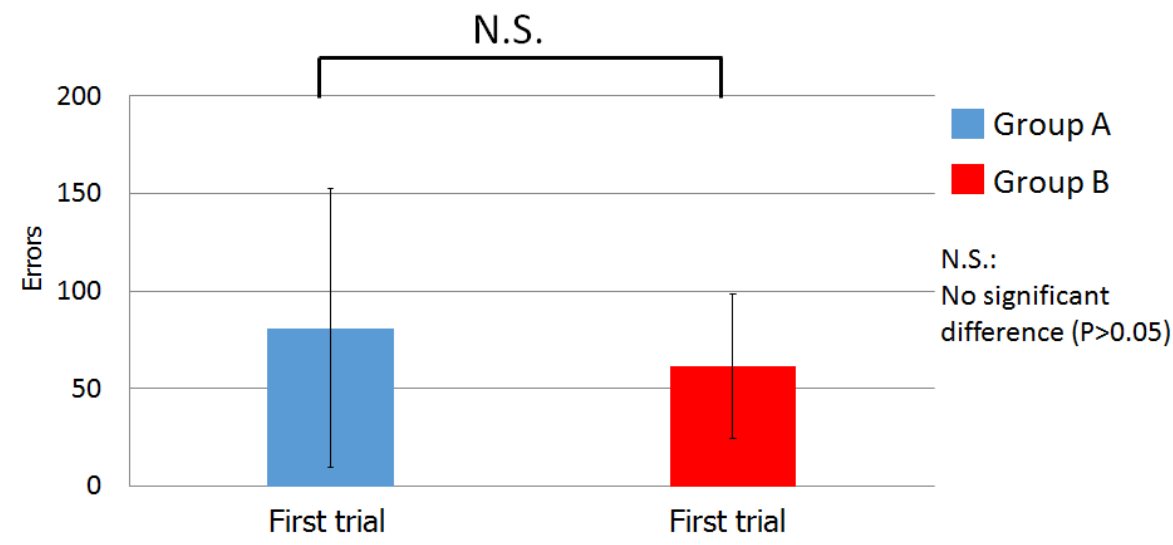

Fig. 13 Comparison between the groups at the first trial

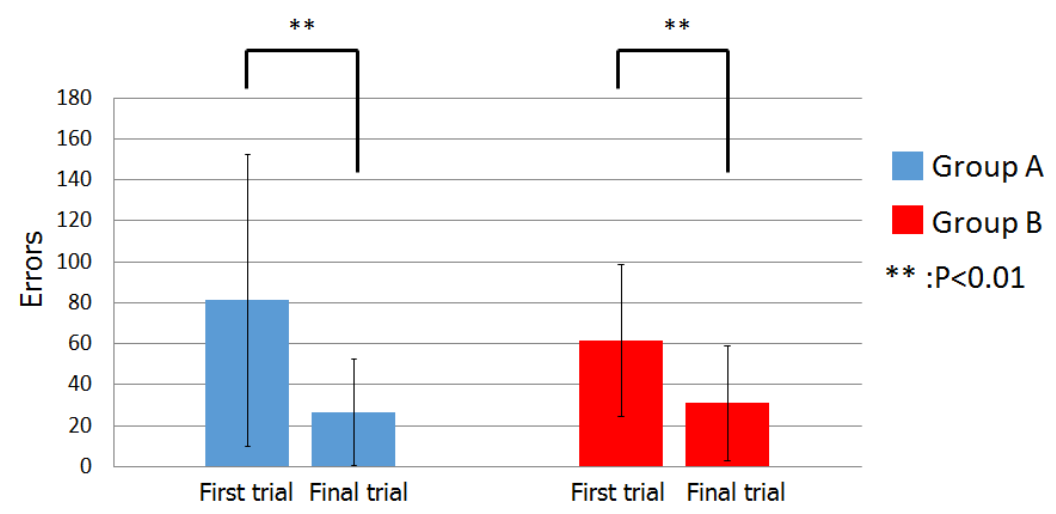

Fig. 14 Comparison between first and final trial

\section{Training Reaction Time (TRT)}

Workload could be deducted from the level of attention and to assess the level of attention on the training, a parameter called Training reaction time (TRT) was used. During the training, the trainee needed to pay attention to the DRT device. When the DRT LED turned on, the trainee needed to press the force sensor which was fixed on the laparoscopic instrument as soon as possible. The reaction time was defined as the elapsed time with the LED ON. However, to compute the real reaction time during laparoscopic training, we should subtract the average subjective baseline measured on the preparation stage. Training reaction time (TRT) was calculated by the formula (2)

$$
\text { Training Reaction Time }(T R T)=\text { Reaction Time }- \text { Baseline }
$$

Fig.15 shows the TRT data for every subject and Fig.16 presents the difference between Group A and Group B. In Fig.16, the TRT of Group B is much higher than Group A. More specifically, in the Group B, we found a high standard deviation for every subject. By analyzing the experiment video, we found that 
the major difference between Group A and Group B was that when the supraliminal visual red cross appeared, trainees needed to complete the training action, but they also were forced to correct the upper limbs posture. These two tasks made the trainees always forgetting to press the force sensor when LED turned on. The trainees in the subliminal group, instead, just had one conscious task, the laparoscopic training, because the subliminal stimulus was transmitted at the subconscious level and did not require attention. They could easily see when the LED turned on and pressed the force sensor immediately. We can deduct from this comparison that the workload during the training was indeed lower for the subliminal stimuli group than for the supraliminal stimuli group, as expected (Hyp 2).

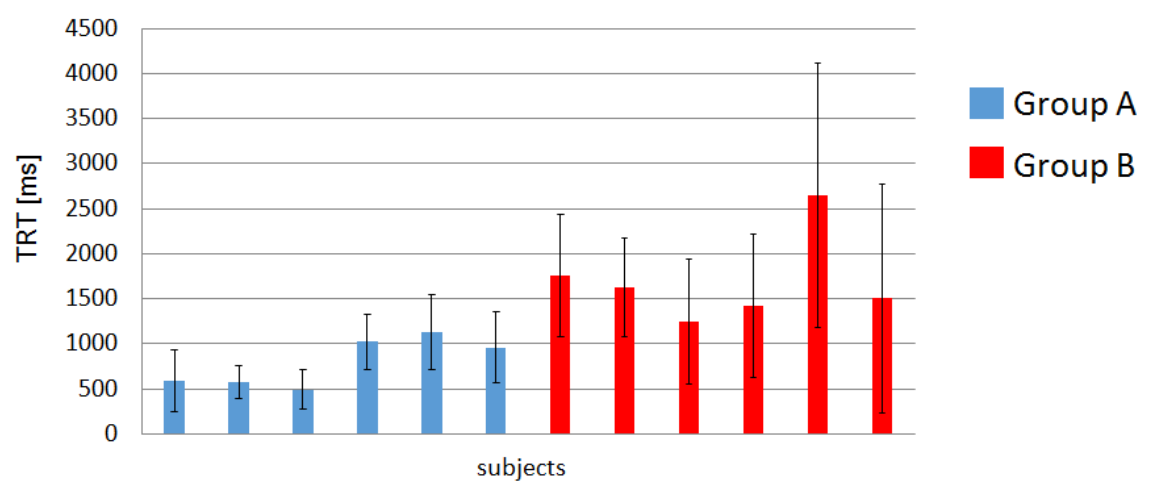

Fig. 15 Training Reaction Time for each subjects

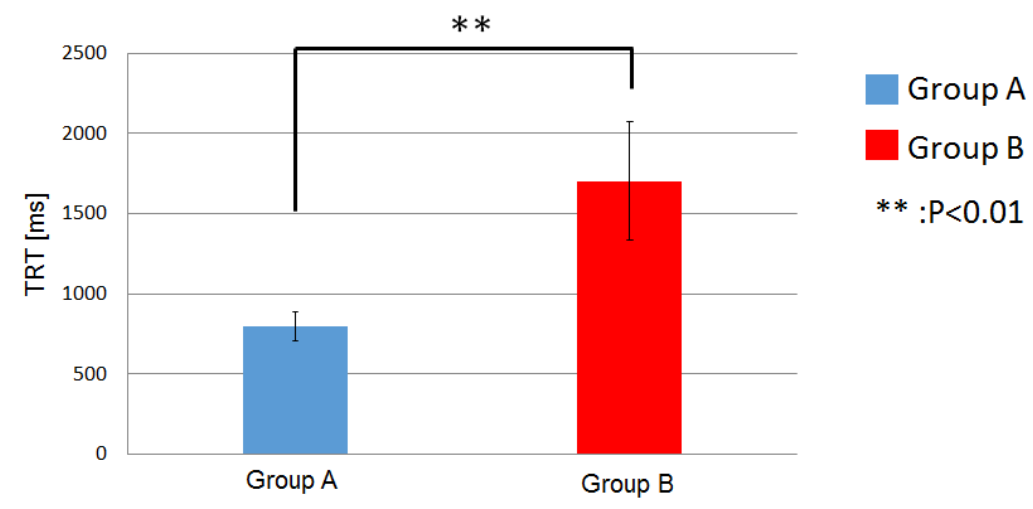

Fig. 16 Training Reaction Time for each subjects

\section{Conclusions}

The current training for laparoscopic practices is focused mainly on enhancing the technical manual skills, without considering the trainee's posture during the training stage. In this work we have proposed a method, namely subliminal persuasion, to give real time stimuli for improving the upper limbs posture during the laparoscopic training. We also confirmed the difference between the stimuli transmitted on the 
conscious level and subconscious level. In fact, we found that subliminal stimuli have the same effect as the supraliminal stimuli improving the posture. However, subliminal stimuli had a lower increment on the workload compared to the supraliminal stimuli. In future work, we want to add a control group without posture feedback for comparison, and also perform experiments involving more difficult and complex laparoscopic training tasks. Moreover, we want to add different distracting stimuli at the same time to simulate a surgical scenario.

A further study in this direction would be to analyze what kind of information should be transmitted on the conscious level or subconscious level. Furthermore, we hope to develop a new training method using different human information processing channels so that the trainee could receive more information during laparoscopic training to improve much faster.

We assume that this method can be used not only for training but also in a real clinical situation. Subliminal persuasion could become an effective method to monitor useful information, such as the surgeon's fatigue and concentration levels, and to recommend resting periods.

\section{Conflict of interests}

The authors declare no conflicts of interest in preparing this article.

\section{Acknowledgments}

This study was partially supported by the research institute of science and engineering, Waseda University. This research has been supported by the JSPS Scientific Research-C grant [24500616], the JSPS Grant-in-Aid for Young Scientists (Wakate B) [25750259], the Waseda University Grant for Special Research Projects (for new full-time faculty) [2014S-091], the Global COE Program "Global Robot Academia", MEXT, Japan, and the Consolidated Research Institute for Advanced Science and Medical Care, Waseda University (ASMeW). It was also partially supported by a grant by STMicroelectronics, which also provided the core sensors and the microcontroller. The authors would like to express their thanks to the Italian Ministry of Foreign Affairs, General Directorate for Cultural Promotion and Cooperation, for its support to RoboCasa. The authors would also like to express their gratitude to Life Performance Research for their support to the research.

References

1. Johnson, A.: Laparoscopic surgery. The Lancet. 349, 631-635 (1997).

2. Novitsky, Y.W., Litwin, D.E.M., Callery, M.P.: The net immunologic advantage of laparoscopic surgery. Surgical Endoscopy. 18, 1411-1419 (2004).

3. Kennedy, G.D., Heise, C., Rajamanickam, V., Harms, B., Foley, E.F.: Laparoscopy Decreases Postoperative Complication Rates After Abdominal Colectomy. Annals of Surgery. 249, 596-601 (2009).

4. Golub, R., Siddiqui, F., Pohl, D.: Laparoscopic Versus Open Appendectomy: A Metaanalysis. 
Journal of the American College of Surgeons. 186, 545-553.

5. Lacy, A.M., García-Valdecasas, J.C., Delgado, S., Castells, A., Taurá, P., Piqué, J.M., Visa, J.: Laparoscopy-assisted colectomy versus open colectomy for treatment of non-metastatic colon cancer: a randomised trial. The Lancet. 359, 2224-2229 (2002).

6. Perrin, M., Fletcher, A.: Laparoscopic abdominal surgery. Contin Educ Anaesth Crit Care Pain. 4, 107-110 (2004).

7. Berguer, R., Smith, W.D., Chung, Y.H.: Performing laparoscopic surgery is significantly more stressful for the surgeon than open surgery. Surgical Endoscopy. 15, 1204-1207 (2001).

8. R. Berguer, M. Remler, D. Beckley: Laparoscopic instruments cause increased forearm fatigue: A subjective and objective comparison of open and laparoscopic techniques. Minimally Invasive Therapy \& Allied Technologies. 6, 36-40 (1997).

9. Tanoue, K., Ieiri, S., Konishi, K., Yasunaga, T., Okazaki, K., Yamaguchi, S., Yoshida, D., Kakeji, Y., Hashizume, M.: Effectiveness of endoscopic surgery training for medical students using a virtual reality simulator versus a box trainer: a randomized controlled trial. Surg Endosc. 22, 985-990 (2008).

10. Zhuohua Lin, Munenori Uemura, Massimiliano Zecca, Salvatore Sessa, Hiroyuki Ishii, Luca Bartolomeo, Kazuko Itoh, Morimasa Tomikawa, Takeshi Odaira, Kazuo Tanoue, Satoshi Ieiri, Kozo Konishi, Makoto Hashizume, Atsuo Takanishi,: Objective Evaluation of Laparoscopic Surgical Skills Using Waseda Bioinstrumentation System WB-3(2010)

11. Corlett EN, Bishop RP : A technique for assessing postural discomfort. Ergonomics 19: 175182,(1976).

12. Bhatnager V, Drury CG, Schiro SG: Posture, postural discomfort,and performance. Hum Factors 27: 189-199, (1985)

13. Kanfer,R.,\& Ackerman, P.: .Motivation and cognitive abilities: Anintegrative aptitude-treatment interaction approach to skill acquisition. Journal of Applied Psychology, 74, 657-690. (1989)

14. Lysaught, R.J., Hill, S.G., Dick, A.O. Plamondon, B. D., Linton, P.M., Wierwille, W. W.,Zaklad, A.L. Bittner, A. C., \&Wherry, R. J.. :Operator workload: Comprehensive review and evaluation of operator workload methodologies. Report No. 851. Willow Grove, PA: Analytics,(1989)

15. Michael A. Cohen, Patrick Cavanagh, Marvin M. Chun and Ken Nakayama.:The attentional requirements of consciousness, Trends in Cognitive Sciences, vol. 16, pp. 411-417,(2012).

16. H. Egermann, R. Kopiez, and C. Reuter: Is there an effect of subliminal messages in music on choice behavior?, J. Articles Support Null Hypothesis, vol. 4, no. 2, pp. 29-46, (2006).

17. D. L. Rosen and S. N. Singh:An investigation of subliminal embed effect on multiple measures of advertising effectiveness, Psychol. Marketing, vol. 9, no. 2, pp. 157-173, (1992).

18. Andreas Riener : Subliminal Persuasion and Its Potential for Driver Behavior Adaptation, TRANSACTIONS ON INTELLIGENT TRANSPORTATION SYSTEM, Vol.13, No.1, (2012) 
19. Lin, Z., Zecca, M., Sessa, S., Bartolomeo, L., Ishii, H., Takanishi, A.: Development of the wireless ultra-miniaturized inertial measurement unit WB-4: Preliminary performance evaluation. 2011 Annual International Conference of the IEEE Engineering in Medicine and Biology Society,EMBC. pp. $6927-6930$ (2011).

20. Lin, Z., Zecca, M., Sessa, S., Bartolomeo, L., Ishii, H., Itoh, K., Takanishi, A.: Development of the miniaturized wireless Inertial Measurement Unit WB-4: Pilot test for mastication analysis. 2010 IEEE/SICE International Symposium on System Integration (SII). pp. 420-425. IEEE (2010).

21. Kantowitz, B. H.. Defining and measuring pilot mental workload. In J. R. Comstock, Jr. (Ed.), Mental state estimation 1987 (pp. 179-188). Hampton, VA: National Aeronautics and Space Administration, Scientificand Technical Information Division, (1988)

22. David L. Strayer, Joel M. Cooper, Jonna Turrill, James Coleman, Nate Medeiros-Ward, and Francesco Biondi: Measuring Cognitive Distraction in the Automobile. (June 2013).

23. Derossis, A.M., Fried, G.M., Abrahamowicz, M., Sigman, H.H., Barkun, J.S., Meakins, J.L.: Development of a Model for Training and Evaluation of Laparoscopic Skills 1. American Journal of Surgery. 175, 482-487.

24. Ritter, E.M., Scott, D.J.: Design of a Proficiency-Based Skills Training Curriculum for the Fundamentals of Laparoscopic Surgery. SURG INNOV. 14, 107-112 (2007). 